\title{
The first case of novel variants of the FSHR mutation causing primary amenorrhea in 2 siblings in Korea
}

\author{
Sukdong Yoo', \\ Ju Young Yoon', \\ Changwon Keum², \\ Chong Kun Cheon ${ }^{1}$ \\ ${ }^{1}$ Department of Pediatrics, Pusan \\ National University School of Medicine, \\ Busan, Korea \\ ³Billion, Inc., Seoul, Korea
}

Follicle-stimulating hormone receptor (FSHR) mutation is a rare cause of amenorrhea. We report the first case of FSHR mutations in Korea. Two female siblings aged 16 (patient 1 ) and 19 (patient 2) years were referred to the pediatric endocrinology clinic because of primary amenorrhea despite normal breast budding. Gonadotropin-releasing hormone stimulation test showed markedly elevated luteinizing hormone and follicle-stimulating hormone with a relatively low level of estrogen, suggesting hypergonadotropic hypogonadism. Pelvic magnetic resonance imaging revealed a bicornuate uterus in patient 1 and uterine hypoplasia with thinning of the endometrium in patient 2 . The progesterone challenge test revealed no withdrawal of bleeding. After 2 months of administration of combined oral contraceptives, menarche was initiated at regular intervals. To determine the genetic cause of amenorrhea in these patients, whole-exome sequencing (WES) was performed and revealed a compound heterozygous FSHR mutation, i.e., c.1364T>G (p.Val455Gly) on exon 10 and c.374T>G (p.Leu125Arg) on exon 4. Both of these were novel mutations and were confirmed by Sanger sequencing. Both patients maintained regular menstruation and improved bone mineral density while taking combined oral contraceptives, calcium, and vitamin D. Therefore, FSHR mutations can be the cause of amenorrhea in Koreans, and WES facilitates the diagnosis of rare causes of amenorrhea.

Keywords: Amenorrhea, Hypogonadism, Follicle-stimulating hormone receptor

\section{Highlights}

- FSHR mutation should be considered a rare cause of delayed puberty, amenorrhea, and hypergonadotropic hypogonadism in Korea.

- The bicornuate uterus and thinning of the endometrium in our case suggested an association between FSHR mutation and pathology in normal uterine development.

\section{Introduction}

Revised: 20 August, 2021

Accepted: 30 August, 2021

Address for correspondence:

Chong Kun Cheon

Department of Pediatrics, Pusan

National University Children's

Hospital, 20 Geumo-ro, Mulgeum-

eup, Yangsan 50612, Korea

Email: chongkun@pusan.ac.kr

https://orcid.org/0000-0002-86095826
Follicle-stimulating hormone (FSH) plays an important role in normal follicular maturation and regulation of estrogen production by granulosa cells in females. ${ }^{1 .}$ The FSH receptor (FSHR) gene (OMIM \#136435) is located on chromosome 2p21 and contains 10 exons spanning 54 $\mathrm{kb}^{2)}$ FSHR mutation can be caused by homozygous or compound heterozygous and activating or inactivating mutations, which are inherited in an autosomal recessive or dominant fashion. ${ }^{1}$ To date, 36 mutations in FSHR have been reported in HGMD (HGMD Professional 2020.4 http://www.hgmd.cf.ac.uk/ac/index.php). Among them, missense mutations (80.5\%) were the most frequent, followed by regulatory mutations (11.1\%). Here, we describe the first Korean cases of FSHR variants manifesting as primary amenorrhea in a pair of siblings. We also 
provide a literature review of inactivating mutations of FSHR in females.

\section{Case report}

Two female siblings aged 16 (patient 1) and 19 (patient 2) years were referred to our pediatric endocrinology clinic because of primary amenorrhea. Secondary sex characteristics were present; Tanner staging of breast/pubic hair was IV/III and III/I for patients 1 and 2, respectively. Breast budding was initiated at the age of 11 years in both patients. The external genitalia was normal in both patients. Anthropometric measurements of patient 1 were within the normal range, but patient 2 had low standard deviation scores for height (-2.31), weight (-2.73), and body mass index (-1.60). Both patients blood pressure was normal. Abnormal physical examinations, including dysmorphic features, hirsutism, and goiter, were not identified. There was no history of strenuous exercise, abnormal eating behavior, or significant weight changes. One month prior to consultation, patient 1 visited a local clinic because of abdominal pain and underwent abdominal computed tomography, which revealed a bicornuate uterus. Otherwise, there was no specific medical history.

The results of laboratory testing were similar between the siblings. Both patients' hemoglobin levels and thyroid function test results were normal, and their urine human chorionic gonadotropin level was negative. Other hormonal results of testosterone, free testosterone, dehydroepiandrosterone sulfate, prolactin, and sex hormone-binding globulin were within normal limits. The anti-Müllerian hormone level in patient 2 was not low. The FSH level was elevated, and subsequent gonadotropin-releasing hormone stimulation testing showed markedly elevated levels of peak luteinizing hormone (LH) and FSH, suggesting hypergonadotropic hypogonadism in both patients. The adrenocorticotropic hormone stimulation test showed a normal response, which enabled exclusion of congenital adrenal hypoplasia in both patients. The karyotypes of them were $46, \mathrm{XX}$ (Table 1 )

Pelvic magnetic resonance imaging (MRI) showed abnormalities in the reproductive organs of both patients. MRI of patient 1 showed a normal bilateral ovary but a bicornuate uterus with a pair of endometrial canals widely separated by the myometrial septum extending to the internal cervical os. Likewise, MRI of patient 2 confirmed a normally shaped but hypoplastic uterus $(1.3 \mathrm{~cm} \times 3.7 \mathrm{~cm})$ with endometrial thinning. In addition, ovarian size, shape, and enhancement were normal in both patients. The vaginal tracts were also normal, and there were no abnormalities associated with the urinary tract or kidneys in either sibling (Fig.

Table 1. Laboratory and hormonal profile of patient with FSHR mutation

\begin{tabular}{|c|c|c|c|}
\hline Variable & Patient 1 ( 16 years old) & Patient 2 (19 years old) & Reference range \\
\hline Free T4 (ng/dL) & 1.01 & 1.01 & $0.7-1.48$ \\
\hline TSH $(\mu \mathrm{lU} / \mathrm{mL})$ & 0.46 & 0.43 & $0.27-4.2$ \\
\hline Renin (ng/mL/hr) & 1.4 & 1.17 & $0.15-2.33$ \\
\hline Aldosterone (ng/dL) & 2.7 & 2.9 & $1.3-14.5$ \\
\hline C-telopeptide (ng/mL) & 1.09 & 0.9 & $\leq 0.57$ \\
\hline Bone ALP (ug/L) & 25.1 & 24.7 & $\leq 14.3$ \\
\hline $\mathrm{LH}(\mathrm{mlU} / \mathrm{mL})$ & 36.87 & 23.39 & $0.5-41.7$ \\
\hline $\mathrm{FSH}(\mathrm{mlU} / \mathrm{mL})$ & 71.79 & 81.26 & $1.5-12.9$ \\
\hline Prolactin (ng/mL) & 4.68 & 10.73 & $3-24$ \\
\hline Estradiol (pg/mL) & 13.56 & 6.37 & $\begin{array}{c}\text { Follicular phase: } 26.6-161 \\
\text { Luteal phase: } 32.7-201\end{array}$ \\
\hline Free testosterone $(\mathrm{pg} / \mathrm{mL})$ & 1.13 & 0.79 & $0-3.09$ \\
\hline Testosterone (ng/mL) & 0.31 & 0.0761 & $0.05-0.4$ \\
\hline SHBG (nmol/L) & 68.9 & 80.5 & $18-144$ \\
\hline DHEA-S ( $\mu \mathrm{g} / \mathrm{dL})$ & 171.1 & 156.4 & 16-189.6 \\
\hline Urine beta-hCG & Negative & Negative & - \\
\hline $\mathrm{AMH}(\mathrm{ng} / \mathrm{mL})$ & Not done & 3.58 & $1.0-4.0$ \\
\hline \multicolumn{4}{|c|}{ ACTH stimulation test (0 min-30 min) } \\
\hline 17-OH-progesterone (ng/mL) & $1.26-2.48$ & $0.38-1.94$ & $0.2-2.65$ \\
\hline ACTH $(p g / m L)$ & $17.39-24.11$ & $4.37-12.08$ & $10-60$ \\
\hline Cortisol ( $\mu \mathrm{g} / \mathrm{dL})$ & $9.95-22.32$ & $2.25-24.52$ & $3-21$ \\
\hline \multicolumn{4}{|l|}{ GnRH stimulation test } \\
\hline LH peak (mIU/mL) & 200 & 200 & $5.5-30$ \\
\hline FSH peak (mIU/mL) & 124 & $>200$ & $4.5-12$ \\
\hline Chromosome & $46, X X$ & $46, X X$ & \\
\hline
\end{tabular}

TSH, thyroid-stimulating hormone; ALP, alkaline phosphatase; LH, luteinizing hormone; FSH, follicle-stimulating hormone; SHBG, sex hormone binding globulin; DHEA-S, dehydroepiandrosterone sulfate; hCG, human chorionic gonadotropin; AMH, anti-Müllerian hormone; $\mathrm{ACTH}$, adrenocorticotropic hormone; $\mathrm{GnRH}$, gonadotropin-releasing hormone. 
1). Bone densitometry showed a low $z$-score $<-2.0$ in the femur neck and lumbar spine, compatible with juvenile osteoporosis. The levels of bone turnover markers, including C-telopeptide and bone alkaline phosphatase, were increased (Table 1).

A progesterone challenge test was performed with medroxyprogesterone $5 \mathrm{mg}$ daily for 10 days. However, withdrawal bleeding was not induced, suggesting inadequate production of endogenous estrogen. Combined oral contraceptives (drospirenone and ethynyl estradiol) were administered. Menarche was initiated at regular intervals after 2 months in both patients.

Whole-exome sequencing (WES) was performed to determine the genetic causes of amenorrhea. Genomic DNA was extracted from the patients' buccal swab samples. All exon regions of all human genes $(22,000)$ were captured using the SureSelect kit (version C2, December 2018; Agilent
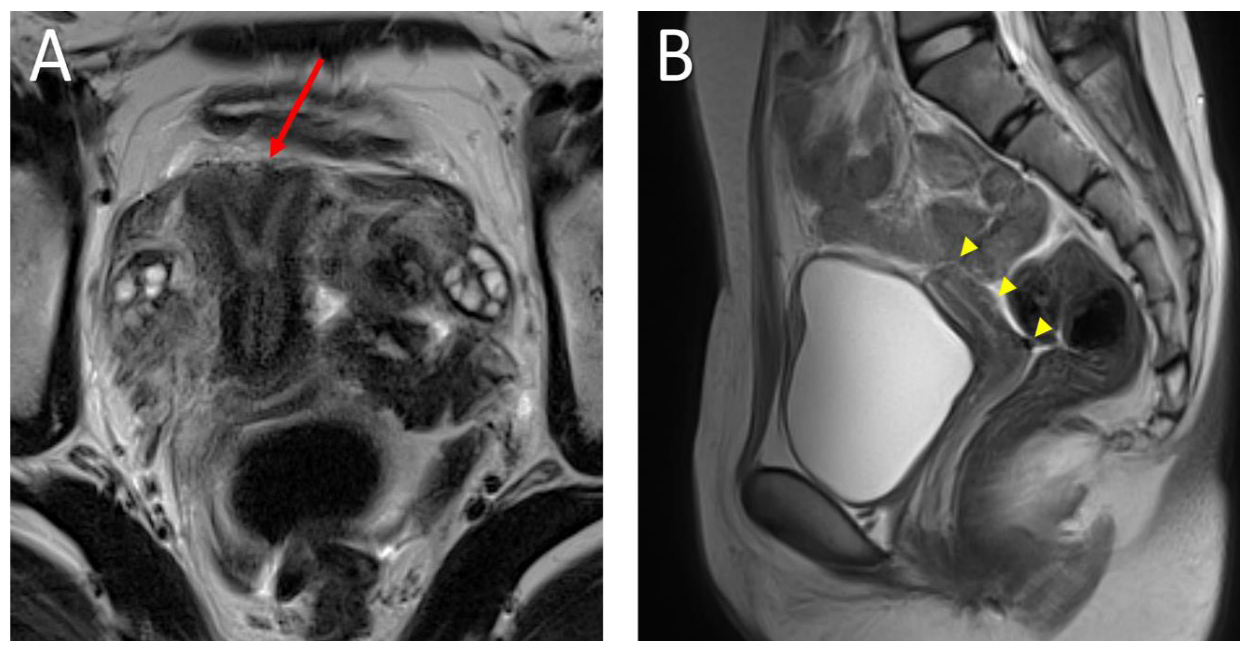

Fig. 1. Pelvic magnetic resonance imaging of patients. (A) Sagittal T1-weighted and coronal oblique T2-weighted BLADE sequence imaging of patient 1 revealing a bicornuate uterus (arrow) with a pair of endometrial canals widely separated by the myometrial septum extending to the internal cervical os. Note the fundal cleft of the outer uterine contour. (B) Sagittal T2 BALDE sequence imaging of patient 2 showed hypoplasia of the uterus $(1.3 \mathrm{~cm} \times 3.7 \mathrm{~cm})$ (arrowheads) with thinning of the endometrium. Size, shape, and enhancement were normal in both ovaries (not seen in this image).

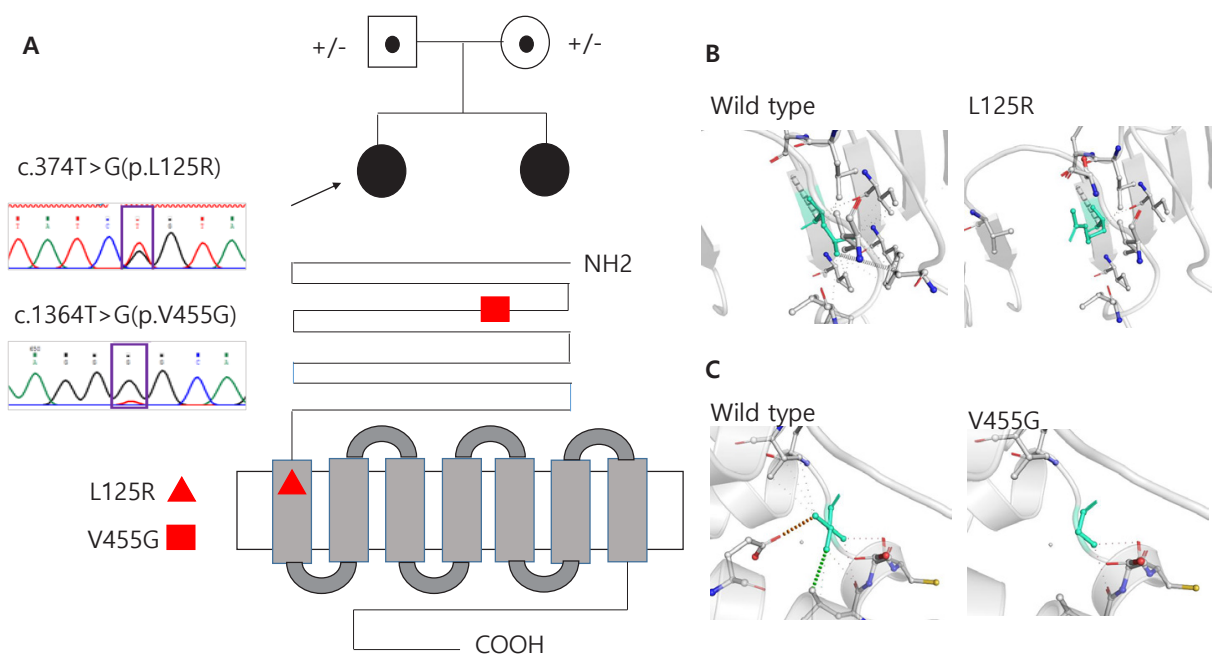

Fig. 2. Two novel heterozygous variants in the FSHR gene. (A) Sanger sequencing confirmed novel variants of c.374T>G (p.L125R) or c.1364T>G (p.V455G) in FSHR in 2 siblings, respectively. L125R is located on the leucine-rich repeats domain, and V455G is located on the transmembrane receptor domain, as shown in the red triangle and square. (B, C) Wild-type and mutant residues (p.L125R and p.V455G) in the FSHR protein are shown in light-green and also are represented as sticks alongside the surrounding residues, indicating any type of interaction. Green dots represent hydrophobic contacts with a surrounding residue. Red dots represent hydrogen bonds with a surrounding residue. The crystal structure of the domain from wild-type FSHR was generated by SWISS-MODEL (https://swissmodel.expasy.org/) and has been depicted as a cartoon representation. All structural images were generated using PyMOL (https://pymol.org/). 
Technologies, Santa Clara, CA, USA) and were sequenced using the NovaSeq platform (Illumina, San Diego, CA, USA). WES revealed a compound heterozygous FSHR mutation, i.e., c.1364T $>$ G (p.Val455Gly) on exon 10 and c.374T $>$ G (p.Leu125Arg) on exon 4; both of these were novel mutations and were confirmed by Sanger sequencing (Fig. 2A). Moreover, both siblings maintained regular menstruation and improved bone mineral densities while taking combined oral contraceptives, calcium, and vitamin D.

This study was approved by the Institutional Review Board (IRB) of Pusan National University Yangsan Hospital (IRB No. 05-2021-045). Informed consent was obtained from the patients for publication and genetic analysis.

\section{Discussion}

FSH is a dimeric glycoprotein composed of $\alpha$ - and $\beta$-subunits released from the anterior pituitary gland. ${ }^{3)}$ This gonadotropin exerts its gonadal and reproductive function by binding to the FSH receptors of testicular sertoli or ovarian granulosa cells. ${ }^{3)}$ Although the early stages of follicular growth occur independently, FSH is essential for granulosa cell differentiation, follicular growth, and oocyte maturation. ${ }^{4)}$

The FSH receptor is a G protein-coupled receptor encoded by the FSHR gene containing 10 exons and 9 introns. ${ }^{2)}$ Exons 1-9 encode the extracellular domain, while exon 10 encodes the C-terminal part of the extracellular domain, the transmembrane domain, and the intracellular domain. ${ }^{2)}$ The extracellular domains are responsible for the specificity and high affinity of ligand binding, whereas the transmembrane domains are responsible for $\mathrm{G}$ protein receptor activation and signal transduction. ${ }^{3)}$ In our case, mutations occurred in exons 4 and 10, which are responsible for extracellular membrane and transmembrane domains, respectively. Two of the variants identified in this study (p.L125R and p.V455G in FSHR) have not been previously reported and were absent in the gnom $\mathrm{AD}$ and 1000 genome population databases. All variants were predicted to be deleterious by a trio of bioinformatics programs (SIFT: 0.00, 0.02; PolyPhen-2: 1.00, 1.00; and MutationTaster: $0.99,0.99)$. Protein crystallization revealed that the p.L125R and p.V455G variants might affect the formation of hydrophobic contacts (Fig. 2B) and that of hydrogen bonds and hydrophobic contacts between the surrounding residues (Fig. $2 \mathrm{C}$ ), respectively. Moreover, due to structural alterations, the p.V455G variant caused changes in vibration-related entropy change upon mutation, increasing molecule flexibility $(\Delta \Delta \mathrm{SVib}$ ENCoM: $0.501 \mathrm{kcal} / \mathrm{mol} / \mathrm{K}$ ), which led to protein destabilization $(\Delta \Delta \mathrm{G}$ : $-0.465 \mathrm{kcal} / \mathrm{mol}$ ) based on structure-based predictions (Supplementary Fig. 1).

Fan and Hendrickson ${ }^{3)}$ demonstrated the nature of FSHFSH receptor interactions through their crystal structure. Each subunit of FSH binds to the concave face of the curved tubeshaped extracellular domain of the FSH receptor in a direction perpendicular to the hand-clasp fashion, which is accompanied by conformational changes in the $\alpha$-subunit loops adjusting their shape to reach optimal interaction. FSH-FSH receptor dimerization is followed by various intracellular signaling pathways through activation of the canonical Gs, adenylyl cyclase, cAMP, and protein kinase A.

Inactivating FSHR mutations cause altered cell surface expression, decreased binding capacity, and impaired signal transduction and receptor expression. This can lead to delayed puberty, hypergonadotropic hypogonadism, primary or secondary amenorrhea, infertility, ovarian dysgenesis, predisposition to sex cord ovarian tumors in female individuals, and impaired spermatogenesis in male individuals. ${ }^{1)}$

Existing studies of inactivating FSHR mutations in female individuals are summarized in Table 2, which was created referring to the report by $\mathrm{He}$ et al. ${ }^{6)}$ An inactivating FSHR mutation was first described in Finnish females in 1996. ${ }^{7)}$ Most variants were located in the extracellular domain, transmembrane domain, and. extracellular loop of the FSH receptor, while one variant $(c .1717 \mathrm{C}>\mathrm{T})$ was located in the intracellular loop. In vitro studies have suggested that these variants disrupt cellular functions such as cAMP production, membrane localization, and FSH binding according to variant. The main clinical feature was hypergonadotropic primary amenorrhea. In some cases, Tanner stage III-IV of the underdeveloped breast was noted even in adulthood. Manifestations of the reproductive structure varied. Although there was one case of a normal fertile woman, most showed small-sized ovaries, ovarian dysgenesis, hypoplastic uterus, or endometrial thinning. However, a dysmorphic uterus, such as seen in our case, has not been reported in previous studies. The 5 studies involving histologic biopsy showed follicular arrest up to the small antral stage.

The bicornuate uterus and thinning of the endometrium in our case suggested an association between FSHR mutation and pathology in normal uterine development. There have been several reports regarding the extragonadal expression of FSHR, suggesting the physiological functions of FSH. Shemesh reported that the gonadotropin receptor showing a dynamic pattern of expression in extragonadal reproductive tissue indicated its substantial role in the molecular autocrineparacrine regulation of the estrous cycle. ${ }^{8)}$ Furthermore, LH receptors are expressed in the endometrium, myometrium, and cervix, but FSH receptors are expressed mainly in the cervix. ${ }^{8)}$ Stilley et al. ${ }^{9}$ summarized the distribution of FSH receptors in extraovarian reproductive tissues using various methods. Although there was a discordance in detection method, FSHR protein was observed in the cervix, endometrium, and myometrium. In pregnant women, FSHR protein was observed in the decidua, placental chorionic villi, umbilical cord, amnion, vascular smooth muscle, and endothelium. ${ }^{9}$ Although these studies support the role of FSH in regulating uterine contractile activity, implantation, and the estrous cycle, they do not demonstrate the role of FSH and its receptors in uterine development. One experimental study conducted on FSHRhaploinsufficient aged mice reported uterine abnormalities, such as a nodular structure, cyst, hypertrophic epithelium, 


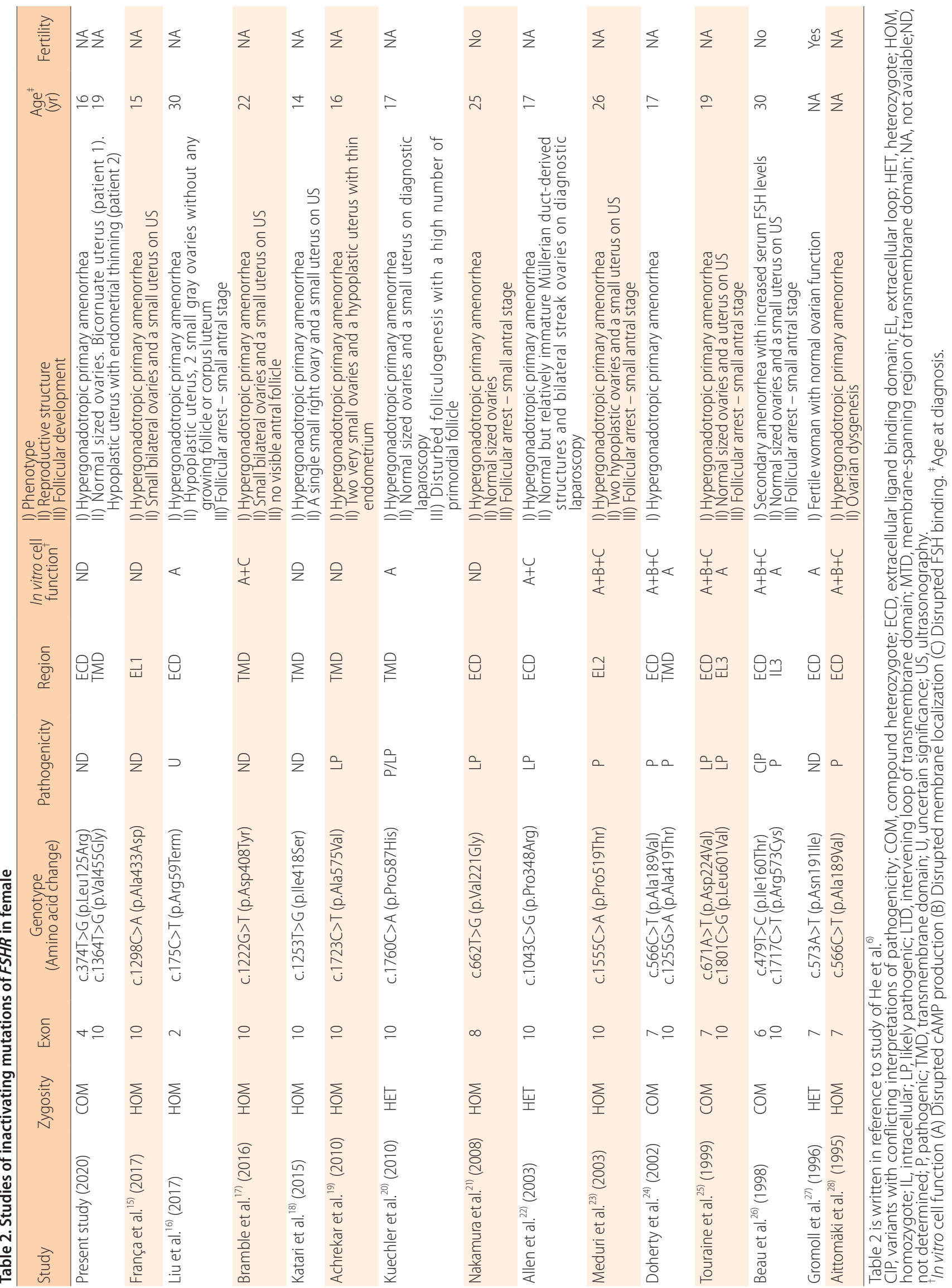


and increased angiogenesis. The authors concluded that an imbalance in progesterone receptor isoforms A and B and increased LH receptors in the uterus may have contributed to a high frequency of uterine pathology. ${ }^{10)}$ Increased gonadotropin in fetuses with FSHR mutation due to gonadal failure and a wide distribution of gonadotropin receptors in reproductive tissues may lead to uterine dysmorphism. However, further research is needed to identify the pathophysiology of the uterus in FSHR mutations.

As with osteoporosis in the 2 siblings in the present case, hypogonadism is highly associated with bone loss. Interestingly, Sun et al. ${ }^{11)}$ showed that neither FSH- $\beta$ nor FSH receptor null mice exhibited bone loss despite severe hypogonadism. In contrast, increased bone mass and decreased osteoclastic resorption were observed in haploinsufficient $\mathrm{FSH}^{+/-}$ mice with normal ovarian function. They concluded that the skeletal action of FSH was estrogen-independent, and high circulating FSH level can cause hypogonadal bone loss. Based on these results, osteoporosis in individuals with FSHR mutation is supposed to be more severe than that in those with hypogonadism without FSH or FSHR pathology. However, further research is required.

Several previous studies conducted in Korea have demonstrated rare prevalence of FHSR mutations. A Korean study comparing infertile and healthy fertile women showed variant types of FSH receptor exon 10 (c.919A>G), but the authors concluded that this mutation may not be specific to premature ovarian failure patients. ${ }^{12)}$ Another Korean study determining the presence of an FSHR mutation (c.566C > T) could not identify any mutation in the FSHR gene in either the premature ovarian failure group or the healthy group. ${ }^{13,14)}$ Therefore, our study is the first report of FSHR mutation causing primary amenorrhea in Korea.

According to our case report, FSHR mutation should be considered a rare cause of amenorrhea in Koreans, and WES gave us the chance to diagnose a rare cause of amenorrhea. Individuals with FSHR mutations can manifest ovarian and uterine pathologies requiring proper radiologic investigation. In addition, they can be more susceptible to osteoporosis than other patients with hypogonadism; therefore, careful management is needed.

\section{Notes}

Supplementary material: Supplementary Fig. 1 can be found at https://doi.org/10.6065/apem.2142116.058.

Conflicts of interest: No potential conflict of interest relevant to this article was reported.

Funding: This study received no specific grant from any funding agency in the public, commercial, or not-for-profit sectors.

Author contribution: Conceptualization: CKC; Data curation: SY, JYY; Formal analysis: SY, CK, CKC; Methodology: CKC; Visualization: CK; Writing - original draft: SY; Writing review \& editing: JYY, CKC

\section{ORCID}

Sukdong Yoo: 0000-0003-2365-9134

Ju Young Yoon: 0000-0002-8317-1192

Chong Kun Cheon: 0000-0002-8609-5826

\section{References}

1. Sperling MA. Sperling pediatric endocrinology E-Book. Amsterdam (The Netherlands): Elsevier Health Sciences, 2020.

2. Simoni M, Gromoll J, Nieschlag E. The follicle-stimulating hormone receptor: biochemistry, molecular biology, physiology, and pathophysiology. Endocr Rev 1997;18:73973.

3. Fan QR, Hendrickson WA. Structure of human folliclestimulating hormone in complex with its receptor. Nature 2005;433:269-77.

4. Richards JS, Pangas SA. The ovary: basic biology and clinical implications. J Clin Invest 2010;120:963-72.

5. Ulloa-Aguirre A, Reiter E, Crépieux P. FSH receptor signaling: complexity of interactions and signal diversity. Endocrinology 2018;159:3020-35.

6. He WB, Du J, Yang XW, Li W, Tang WL, Dai C, et al. Novel inactivating mutations in the $\mathrm{FSH}$ receptor cause premature ovarian insufficiency with resistant ovary syndrome. Reprod Biomed Online 2019;38:397-406.

7. Aittomäki K, Herva R, Stenman UH, Juntunen K, Ylöstalo P, Hovatta $\mathrm{O}$, et al. Clinical features of primary ovarian failure caused by a point mutation in the follicle-stimulating hormone receptor gene. J Clin Endocrinol Metab 1996;81:3722-6.

8. Shemesh M. Actions of gonadotrophins on the uterus. Reproduction 2001;121:835-42.

9. Stilley JAW, Segaloff DL. FSH actions and pregnancy: looking beyond ovarian FSH receptors. Endocrinology 2018;159:4033-42.

10. Danilovich N, Roy I, Sairam MRJE. Emergence of uterine pathology during accelerated biological aging in FSH receptor-haploinsufficient mice. Endocrinology 2002;143:3618-27.

11. Sun L, Peng Y, Sharrow AC, Iqbal J, Zhang Z, Papachristou DJ, et al. FSH directly regulates bone mass. Cell 2006;125:247-60.

12. Kim NK, Lee SH, Nam YS, Sohn TJ, Park SH, Park C, et al. Molecular variants of the FSH receptor exon 10 (Thr307Ala; A919G) in premature ovarian failure (POF) women by PCR-SSCP. Korean J Obstet Gynecol 2000;43:1144-6.

13. Choi YM, Kim SH, Kim JK, Moon SY, Lee JY, Lee GW. Follicle stimulating hormone receptor gene mutation in Korean women with premature ovarian failure and normal karyotype. Korean J Obstet Gynecol 2001;43:836-41.

14. Nam Y, Kim N, Choi M, Park S, Chung K, Lee S, et al. Analysis of follicle stimulating hormone receptor gene mutation in Korean. Clin Exp Reprod Med 1998;25:281-6. 
15. França MM, Lerario AM, Funari MFA, Nishi MY, Narcizo AM, de Mello MP, et al. A novel homozygous missense FSHR variant associated with hypergonadotropic hypogonadism in two siblings from a Brazilian family. Sex Dev 2017;11:137-42.

16. Liu H, Xu X, Han T, Yan L, Cheng L, Qin Y, et al. A novel homozygous mutation in the FSHR gene is causative for primary ovarian insufficiency. Fertil Steril 2017;108:10505.e2.

17. Bramble MS, Goldstein EH, Lipson A, Ngun T, Eskin A, Gosschalk JE, et al. A novel follicle-stimulating hormone receptor mutation causing primary ovarian failure: a fertility application of whole exome sequencing. Hum Reprod 2016;31:905-14.

18. Katari S, Wood-Trageser MA, Jiang H, Kalynchuk E, Muzumdar R, Yatsenko SA, et al. Novel Inactivating Mutation of the FSH Receptor in Two Siblings of Indian Origin With Premature Ovarian Failure. J Clin Endocrinol Metab 2015;100:2154-7.

19. Achrekar SK, Modi DN, Meherji PK, Patel ZM, Mahale SD. Follicle stimulating hormone receptor gene variants in women with primary and secondary amenorrhea. J Assist Reprod Genet 2010;27:317-26.

20. Kuechler A, Hauffa BP, Köninger A, Kleinau G, Albrecht B, Horsthemke B, et al. An unbalanced translocation unmasks a recessive mutation in the follicle-stimulating hormone receptor (FSHR) gene and causes FSH resistance. Eur J Hum Genet 2010;18:656-61.

21. Nakamura Y, Maekawa R, Yamagata Y, Tamura I, Sugino $\mathrm{N}$. A novel mutation in exon8 of the follicle-stimulating hormone receptor in a woman with primary amenorrhea. Gynecol Endocrinol 2008;24:708-12.
22. Allen LA, Achermann JC, Pakarinen P, Kotlar TJ, Huhtaniemi IT, Jameson JL, et al. A novel loss of function mutation in exon 10 of the FSH receptor gene causing hypergonadotrophic hypogonadism: clinical and molecular characteristics. Hum Reprod 2003;18:251-6.

23. Meduri G, Touraine P, Beau I, Lahuna O, Desroches A, Vacher-Lavenu MC, et al. Delayed puberty and primary amenorrhea associated with a novel mutation of the human follicle-stimulating hormone receptor: clinical, histological, and molecular studies. J Clin Endocrinol Metab 2003;88:3491-8.

24. Doherty E, Pakarinen P, Tiitinen A, Kiilavuori A, Huhtaniemi I, Forrest S, et al. A novel mutation in the FSH receptor inhibiting signal transduction and causing primary ovarian failure. J Clin Endocrinol Metab 2002;87:1151-5.

25. Touraine P, Beau I, Gougeon A, Meduri G, Desroches A, Pichard C, et al. New natural inactivating mutations of the follicle-stimulating hormone receptor: correlations between receptor function and phenotype. Mol Endocrinol 1999; 13:1844-54.

26. Beau I, Touraine P, Meduri G, Gougeon A, Desroches A, Matuchansky C, et al. A novel phenotype related to partial loss of function mutations of the follicle stimulating hormone receptor. J Clin Invest 1998;102:1352-9.

27. Gromoll J, Simoni M, Nordhoff V, Behre HM, De Geyter C, Nieschlag E. Functional and clinical consequences of mutations in the FSH receptor. Mol Cell Endocrinol 1996;125:177-82.

28. Aittomäki K, Lucena JL, Pakarinen P, Sistonen P, Tapanainen J, Gromoll J, et al. Mutation in the folliclestimulating hormone receptor gene causes hereditary hypergonadotropic ovarian failure. Cell 1995;82:959-68. 